\title{
Disfunção Ventricular e do Átrio Esquerdo Subclínica em Pacientes com Acromegalia: Um Estudo de Ecocardiograma de Rastreamento de Manchas
}

\author{
Subclinical Left Atrial and Ventricular Dysfunction in Acromegaly Patients: A Speckle Tracking \\ Echocardiography Study
}

\author{
Hasan Koca, ${ }^{\circledR}$ Mevlüt Koc, ${ }^{2}$ Hilmi Erdem Sumbul, ${ }^{3}$ Yahya Kemal Icen, ${ }^{1}$ Erdinc Gulumsek, ${ }^{3}{ }^{\circledR}$ Fadime Koca, ${ }^{4}$ \\ Huseyin Ali Ozturk, ${ }^{3}$ Ahmet Oytun Baykan, ${ }^{5}$ Onur Kaypakli6 ${ }^{(0)}$ \\ Adana Health Practice and Research Center - Department of Cardiology, ${ }^{1}$ Adana - Turquia \\ Adana Numune Training and Research Hospital, ${ }^{2}$ Adana - Turquia \\ Adana Health Practice and Research Center - Department of Internal Medicine, ${ }^{3}$ Adana - Turquia \\ University of Health Sciences - Adana Health Practice and Research Center, ${ }^{4}$ Adana -Turquia \\ Ortadogu Private Health Hospital - Department of Cardiology, ${ }^{5}$ Adana - Turquia \\ Mustafa Kemal University Tayfur Ata Sokmen Faculty of Medicine - Department of Cardiology, ${ }^{6}$ Hatay - Turquia
}

\section{Resumo}

Fundamento: Embora se saiba que a fração de ejeção (FE) do ventrículo esquerdo (VE) medida por eletrocardiograma seja preservada em pacientes com acromegalia, não há informação suficiente sobre deformação longitudinal global e deformação do átrio esquerdo (SLG-VE e SAE).

Objetivo: O objetivo deste estudo foi avaliar as funções do ventrículo esquerdo (VE) e do átrio esquerdo (AE) por ecocardiograma strain (ES) em pacientes com acromegalia.

Métodos: Este estudo incluiu 50 pacientes com acromegalia na forma ativa da doença e 50 controles saudáveis com idade, sexo e área de superfície corporal similares. Além dos ecocardiogramas de rotina, medições de SLG-VE e SAE foram realizadas com o ES.

Resultados: Os valores dos SAE e SLG-VE foram significativamente mais baixos em pacientes com acromegalia ( $p<0,05$ para todos). Na análise bivariada, a pressão arterial sistólica, o pró-hormônio $\mathrm{N}$-terminal do peptídeo natriurético cerebral, o fator de crescimento semelhante à insulina tipo 1, e detectou-se que os níveis de IMVE tinham correlação positiva com SAE e SLG-VE $(p<0,05)$. O nível de IGF-1 tinha forte correlação com SAE e SLG-VE ( $p<0,001$ e $\beta=0,5$ vs. $p<0,001$ e $\beta=0,626$, respectivamente); $48 \%$ dos pacientes com acromegalia têm SLG-VE reduzido ( $<20 \%$ ). $O$ índice de massa do ventrículo esquerdo (IMVE) determina independentemente a presença de SLG-VE reduzido, e cada $1 \mathrm{~g} / \mathrm{m}^{2} \mathrm{de}$ aumento no nível de IMVE aumenta a probabilidade de redução de SLG-VE em $6 \%$.

Conclusão: Embora a fração de ejeção de VE seja normal em pacientes com acromegalia, os valores de SAE e SLG-VE são significativamente mais baixos. Além do aumento em IMVE, outro achado do envolvimento cardíaco pode ser a redução de SAE e SLG-VE. Portanto, além do ecocardiograma de rotina, SAE e SLG-VE podem ser úteis para avaliar os sinais iniciais de envolvimento cardíaco antes da ocorrência de alterações cardíacas irreversíveis.

Palavras-chave: Ecocardrgiografia/métodos; Acromegalia; Doenças Crônicas; Doenças Cardiovasculares; Deformação do Miocárdio; Diagnóstico por Imagem; Volume Sistólico.

\section{Abstract}

Background: Although it is known that the left ventricular (LV) ejection fraction (EF) measured by echocardiography is preserved in patients with acromegaly, there is not enough information about the LV and left atrial strain (LV-GLS and LAS).

Objective: This study aimed to evaluate the left ventricular (LV) and left atrial (LA) functions with strain echocardiography (SE) in patients with acromegaly

Methods: This study included 50 acromegaly patients with active disease and 50 healthy controls with similar age, gender, and body surface area. In addition to routine echocardiography examinations, LV-GLS and LAS measurements were performed with SE.

Correspondência: Hasan Koca •

Adana Health Practice and Research Center - Department of Cardiology - Dr. Mithat Özsan Bulvarı Kışla Mah, 4522 Sok. No: 1 Yüreğir, Adana 01230 - Turquia E-mail: hasankoca90@hotmail.com

Artigo recebido em 04/11/2020, revisado em 11/03/2021, aceito em 28/04/2021

DOI: https://doi.org/10.36660/abc.20201174 
Results: LAS and LV-GLS values were significantly lower in patients with acromegaly ( $<<0.05$ for all). In bivariate analysis, systolic blood pressure, N-terminal prohormone of brain natriuretic peptide, Insulin-like growth factor-1, LA diastolic diameter, and LVMI levels were found to be positively correlated with both LAS and LV-GLS $(p<0.05)$. IGF-1 level was strongly correlated with LAS and LV-GLS $(p<0.001$ and $\beta=0.5$ vs. $p<0.001$ and $\beta=0.626$, respectively); $48 \%$ of patients with acromegaly have reduced LV-GLS ( $<20 \%$ ). Left ventricular mass-index (LVMI) independently determines the presence of reduced $L V$-GLS and each $1 \mathrm{~g} / \mathrm{m}^{2}$ increase in $L V M I$ level increases the likelihood of reduced $L V$-GLS by $6 \%$.

Conclusion: Although LV ejection fraction is normal in patients with acromegaly, LAS and LV-GLS values were significantly reduced. Apart from LVMI increase, another finding of cardiac involvement may be LAS and LV-GLS decrease. Therefore, in addition to routine echocardiography, LAS and LV-GLS may be useful to evaluate early signs of cardiac involvement before the occurrence of irreversible cardiac changes.

Keywords: Echocardiography/methods; Acromegaly; Cardiovascular Diseases; Myocardial, Deformability; Diagnostic,Imaging; Stroke Volume

Full texts in English - http://www.arquivosonline.com.br

\section{Introdução}

A acromegalia é uma doença crônica caracterizada pelo aumento da síntese do fator de crescimento semelhante à insulina tipo 1 (IGF-1) no fígado devido a um adenoma pituitário secretor de hormônio de crescimento $(\mathrm{GH})$, e síntese excessiva de proteína e crescimento tecidual excessivo devido a esses hormônios. ${ }^{1}$ Níveis altos de IGF-1 crônicos causam alterações estruturais e funcionais específicas. ${ }^{1}$ Se não forem tratados, levam à morte, cuja causa mais comum são as doenças cardiovasculares (CV). ${ }^{1,2}$

Strain é uma medida de deformação em relação a uma potência em uma substância. Ele é definido em dimensões radiais, circunferenciais e longitudinais. A avaliação da deformação miocárdica por ecocardiograma strain (ES), em termos de um ecocardiograma de rastreamento de manchas bidimensional (2D-STE) ou imagem por Doppler tecidual (strain convencional) pode oferecer informações incomparáveis sobre as funções ventriculares regionais e globais. ${ }^{3}$ Imagens de deformação podem detectar até as mínimas alterações funcionais e garantir um diagnóstico em fase inicial. Os parâmetros de deformação do átrio esquerdo (AE) e deformação longitudinal global do ventrículo esquerdo (SLG-VE) demonstraram estar fortemente correlacionados às funções sistólicas do $\mathrm{AE}$ e do VE em cenários clínicos diferentes, respectivamente. ${ }^{4-8}$ Demonstrou-se que muitos pacientes com fração de ejeção de VE (FEVE) normal têm redução da função sistólica do VE com o uso do SLG-VE. 3,9,10

Em pacientes com acromegalia, a função sistólica é avaliada com FEVE. Entretanto, deficiências em FEVE só são vistas em etapas posteriores da doença e na minoria dos pacientes. ${ }^{11-14}$ Recentemente, um número limitado de estudos avaliou a deformação de AE e SLG-VE em pacientes com acromegalia e FEVE preservada. ${ }^{5-8}$ Foram obtidos resultados contraditórios nesses estudos. Detectou-se, em dois estudos conduzidos pelos mesmos autores, a redução do SLG-VE; ;,9 enquanto outro estudo relatou que o SLG-VE era semelhante ao dos pacientes de controle saudáveis. ${ }^{5}$ A avaliação das medidas de deformação do AE em pacientes com acromegalia foi realizada com 3D-STE em um estudo; entretanto, não se obtiveram informações claras sobre a alteração da deformação global no AE. ${ }^{8}$

Devido à viabilidade de medições simultâneas da deformação de AE e SLG-VE, além do ecocardiograma tradicional, este estudo teve o objetivo de avaliar as funções do VE e do AE com ES em pacientes com acromegalia ativa e FEVE preservada.

\section{Métodos}

\section{População do estudo}

Neste estudo transversal, 50 pacientes (33 do sexo masculino, 17 do sexo feminino; média de idade de 46,1 $\pm 6,2$ anos) com acromegalia ativa (1-Pacientes recorrentes, 2-Pacientes pós-cirurgia sem remissão, 3-Pacientes em tratamento médico sem remissão) e idade, sexo, índice de massa corporal (IMC) e área de superfície corporal (ASC) correspondentes a 50 pacientes de controle saudáveis (31 do sexo masculino, 19 do sexo feminino; média de idade: 44,6 \pm 5,1 anos). Pacientes acima dos 18 anos de idade com acromegalia ativa foram cadastrados no estudo. Os pacientes incluídos no estudo são apresentados no fluxograma (Figura 1). Informações de diretrizes atuais foram utilizadas para o diagnóstico, o tratamento e a classificação de pacientes com acromegalia. ${ }^{1}$ A remissão da acromegalia foi definida como um $\mathrm{GH}$ sérico suprimido por glicose abaixo de $0,38 \mu \mathrm{g} /$ /itro ( $<1 \mathrm{mU} /$ litro), um $\mathrm{GH}$ sérico abaixo de 1,9 $\mu \mathrm{g} /$ litro $<5 \mathrm{mU} /$ litro), e IGF-1 normal para a idade. ${ }^{1}$ Pacientes com histórico de doença arterial coronariana (DAC) e infarto do miocárdio, arritmia cardíaca, insuficiência cardíaca sistólica ou FEVE $<50 \%$, doença de válvula cardíaca, embolia pulmonar, disfunção da tireoide, gravidez (comprovada ou suspeita), malignidade, e disfunção renal e hepática, e pacientes que se recusaram a participar do estudo não foram incluídos. O comitê de ética local aprovou o protocolo do estudo (Comitê de Ética da Faculdade de Medicina da Universidade Çukurova, 03.05.2019-88), e foi obtido o consentimento informado por escrito de cada um dos participantes.

Após a avaliação do histórico médico detalhado e um exame físico completo, as características de linha de base dos pacientes, incluindo idade, sexo, hipertensão (HT), diabetes mellitus (DM), hiperlipidemia, tabagismo atual, histórico familiar de doenças cardíacas e medicamentos, foram registradas para todos os pacientes. Os parâmetros de IMC e ASC dos participantes foram calculados.

Os níveis de glicose, nitrogênio ureico sanguíneo, creatinina, colesterol total, colesterol de lipoproteína de baixa densidade, colesterol de lipoproteína de alta densidade, triglicérides, aspartato aminotransferase, alanina aminotransferase, leucócitos, hemoglobina, proteína C-reativa sensível alta, e peptídeo natruriético pró-cérebro N-terminal (NT-proBNP) foram medidos utilizando-se um analisador químico automático (Abbott Aeroset, MN, EUA) com os kits 


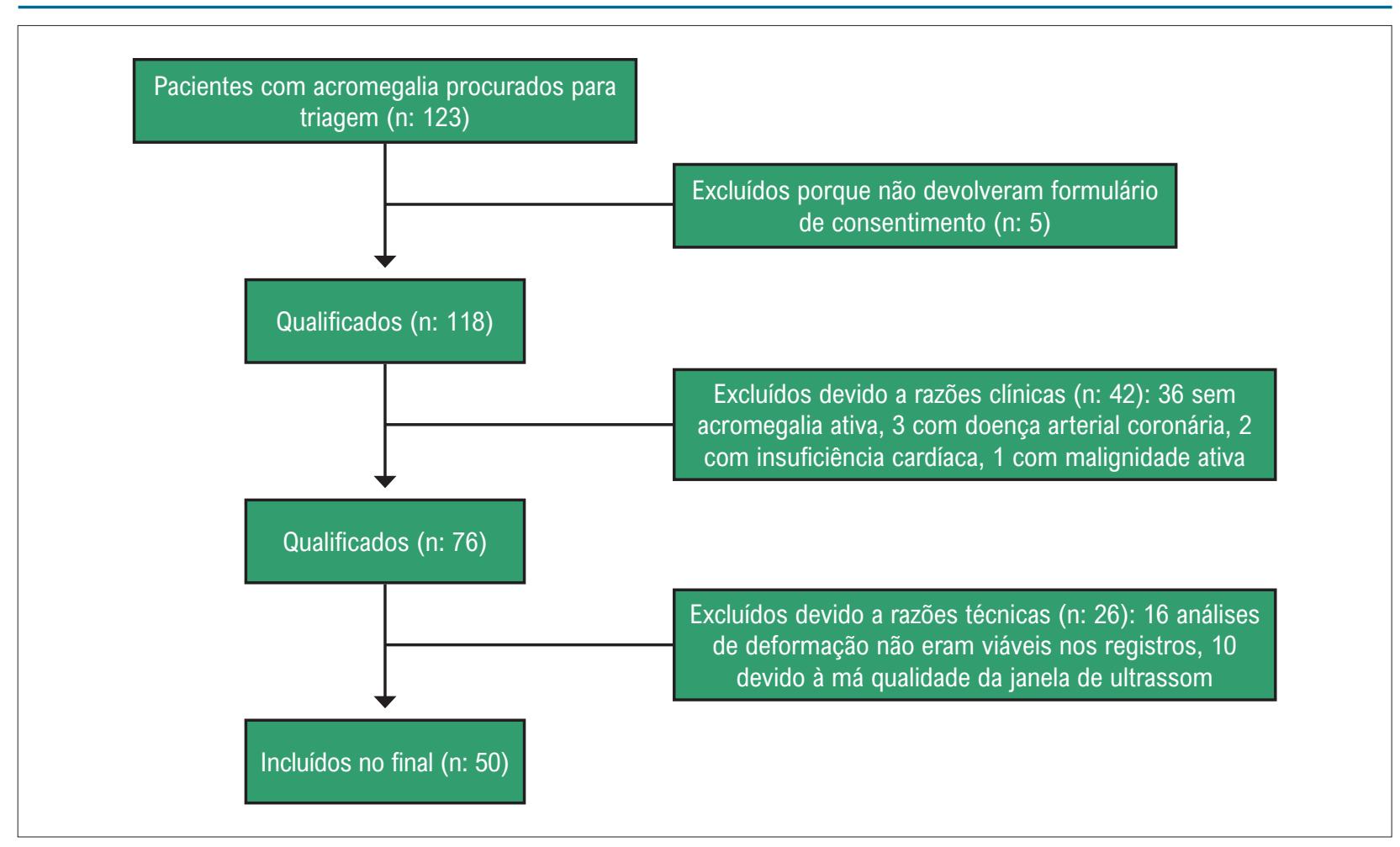

Figura 1 - Fluxograma para inclusão e avaliação de pacientes no estudo.

comerciais apropriados (Abbott). O GH sérico foi avaliado por um analisador químico automático (Abbott Aeroset, MN, EUA) utilizando-se os kits comerciais apropriados (Abbott) e o valor de referência do $\mathrm{GH}$ estava entre $0,014-5,219 \mathrm{ng} / \mathrm{ml}$. O IGF-1 sérico total foi avaliado por um analisador químico automático (Abbott Aeroset, MN, EUA) utilizando-se os kits comerciais apropriados (Abbott) e o valor de referência do IGF1 varia de acordo com idade e sexo. Os níveis de GH e IGF-1 foram medidos ao mesmo tempo do exame ecocardiográfico para cada um dos sujeitos.

\section{Avaliação ecocardiográfica}

A avaliação ecocardiográfica foi feita utilizando-se um transdutor de 2,5-3,5 MHz EPIQ 7C (Philips Healthcare 3000 Minuteman Road, Andover, MA, EUA). A avaliação ecocardiográfica foi realizada durante a primeira semana para pacientes que atenderam aos critérios de inclusão. Avaliações ecocardiográficas de todos os pacientes foram realizadas em posição de decúbito lateral esquerdo com monitoramento de pressão arterial e eletrocardiográfico. Todas as imagens foram obtidas em pelo menos 3 ciclos repetitivos do eixo paraesternal longo e curto padrão, cortes apicais de 4 câmaras, de 5 câmaras, e 2 câmaras de acordo com as sugestões da American Society of Echocardiography (Sociedade Americana de Ecocardiografia). ${ }^{15} \mathrm{O}$ diâmetro diastólico do VE, o diâmetro sistólico do VE, a espessura do septo interventricular (SIV), a espessura da parede posterior (PP), e o diâmetro diastólico do AE foram medidos a partir da imagem do eixo longo paraesternal de janelas de imagem bidimensionais. A fórmula de Devereux foi utilizada para a medição da massa do VE. ${ }^{16}$ Em seguida, o índice de massa do VE (IMVE) foi calculado dividindo-se a massa do VE pela ASC. O valor do IMVE > $115 \mathrm{gr} / \mathrm{m}^{2}$ em homens e >95 gr/m² em mulheres foram considerados hipertrofia do VE. ${ }^{17}$

No procedimento de ES, todos os pacientes tinham ritmo sinusal normal. Os parâmetros de deformação miocárdica de AE e VE foram calculados utilizando-se STE sobre imagens em escala de cinza bidimensionais. Foram registradas imagens apicais de 4 câmaras (A4C), apicais de 2 câmaras (A2C) e apicais de 3 câmaras (A3C) em escala de cinza bidimensionais após a expiração após prender o fôlego. Pelo menos três ciclos cardíacos foram registrados para cada imagem, e prestou-se atenção para se considerar pelo menos 60-80 fps, de acordo com as diretrizes da European Society of Cardiology (Sociedade Europeia de Cardiologia). ${ }^{18}$ Segmentos com qualidade de imagem insuficiente e ciclos cardíacos contendo batimentos prematuros foram excluídos das medições.

O software QLAB versão 10.5 (Philips, Andover, MA, EUA) foi utilizado para as análises de VE e AE. O software seguiu automaticamente os movimentos da parede durante todo o ciclo cardíaco após o endocárdio do VE ter sido marcado quadro a quadro pelo método de desenho manual (rastreamento manual) nas imagens bidimensionais. Os valores de SLG-VE foram calculados a partir das imagens com 2D-STE. Após a marcação manual de 2 partes basais e 1 parte apical do VE, as bordas endocárdicas restantes foram automaticamente marcadas pelo software e a borda epicárdica apropriada também foi automaticamente desenhada. Quando os contornos de VE desenhados automaticamente não eram adequados para análise, as 
bordas foram corrigidas manualmente para permitir uma análise adequada. Após a análise, o software dividiu os registros de $\mathrm{A} 2 \mathrm{C}, \mathrm{A} 3 \mathrm{C}, \mathrm{A} 4 \mathrm{C}$ do $\mathrm{VE}$ em modelos de seis segmentos, e o modelo de 18 segmentos foi usado para calcular o SLG-VE (Figura 2).

Parâmetros de deformação miocárdica do AE também foram calculados a partir das imagens 2D-STE utilizando-se o software da deformação do VE. ${ }^{19}$ Foram obtidas imagens do AE em corte de quatro câmeras utilizando-se marcos anatômicos-padrão para garantir a aquisição ideal e evitar o encurtamento com ecocardiograma bidimensional convencional, em índices de quadros relativamente altos (60-80 fps). O contorno endocárdico do AE foi iniciado na borda endocárdica do anel mitral até a borda endocárdica do $A E$, extrapolando pelas veias pulmonares, e/ou orifícios de apêndices de $\mathrm{AE}$, até o anel mitral oposto por um radiologista experiente, cego em relação às informações clínicas. O software então gerou automaticamente uma silhueta de $\mathrm{AE}$ epicárdica, que delineou uma região de interesse em cada um dos cortes apicais. O ajuste manual da região de interesse foi permitido para incluir toda a camada miocárdica do $A E$, seguido de um rastreamento segmentar automático. Após o rastreamento, os índices de deformação do $\mathrm{AE}$, tais como da deformação longitudinal e suas primeiras curvas SR de derivação foram obtidas a partir de uma vista apical de quatro câmaras. ${ }^{20}$ Utilizamos uma onda R como ponto de partida (ligação R-R) para análise da deformação. A deformação longitudinal e as curvas de índice de deformação foram geradas em todos os segmentos, e a média dos segmentos foi calculada para os pontos de tempo correspondentes (Figura 3). Utilizando essas curvas, a deformação do AE (SAE) e os índices de deformação de pico sistólico (RSAE) foram calculados. O SAE e o RSAE representam a função de reservatório do AE. Todas as imagens ecocardiográficas foram armazenadas digitalmente e analisadas off-line, com as medidas de deformação realizadas por um cardiologista experiente, cego em relação aos dados, utilizando análise de software Philips QLAB versão 10.5.
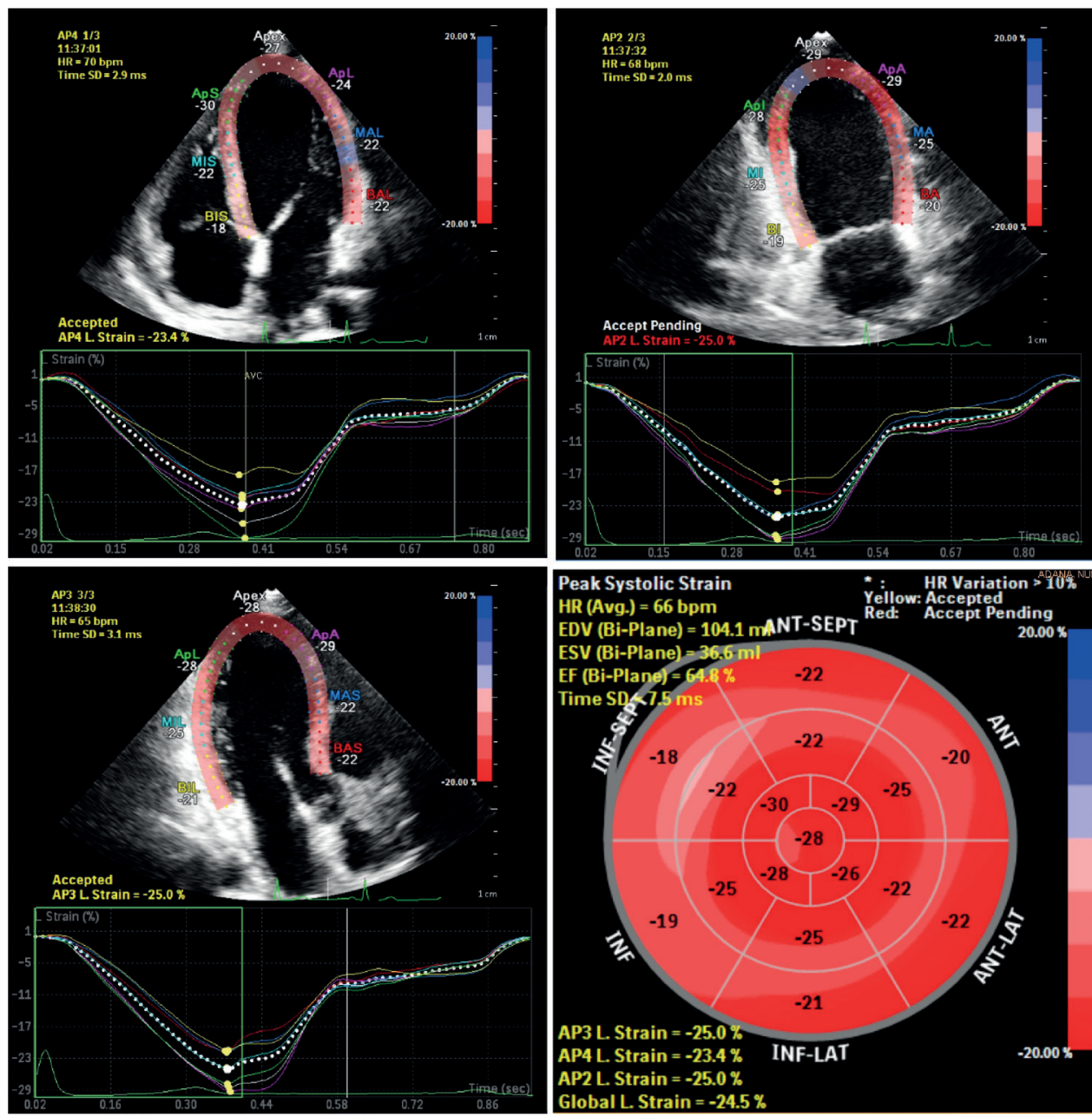

Figura 2 - Medição da deformação longitudinal global do ventrículo esquerdo (SLG-VE) por ecocardiograma strain em pacientes com acromegalia. 


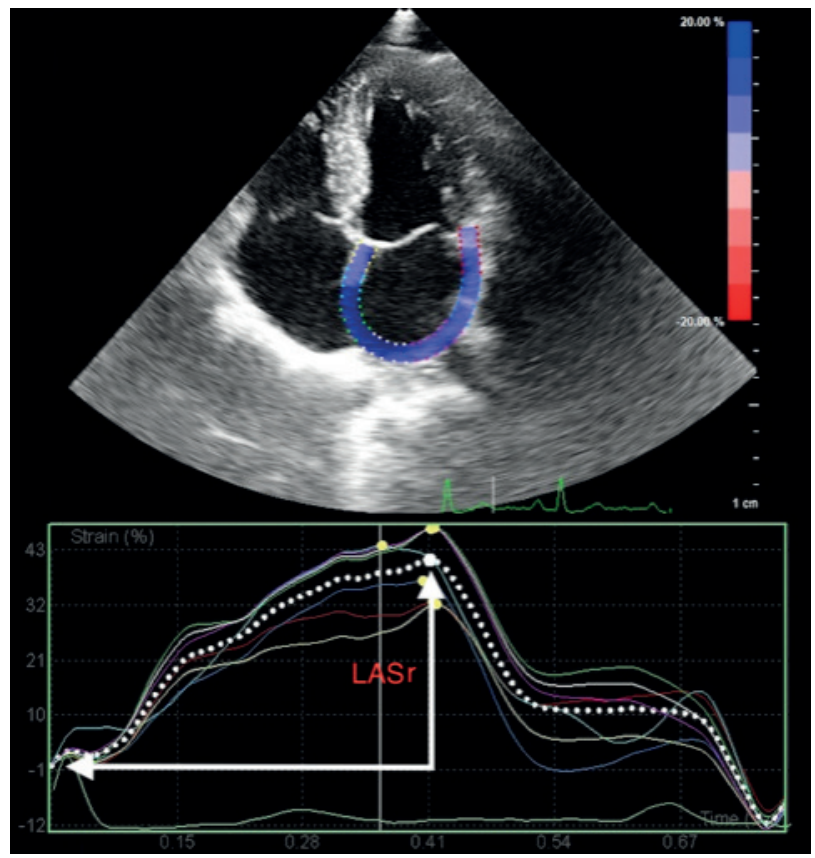

Figura 3 - Medição da deformação longitudinal global do ventrículo esquerdo (SLG-VE) por ecocardiograma strain em pacientes com acromegalia.

\section{Análises estatísticas}

As análises estatísticas foram realizadas utilizando-se o software SPSS, versão 23.0 (SPSS Inc., Chicago, Illinois, EUA). Os dados foram expressos como média \pm DP para variáveis contínuas e porcentagens para variáveis categóricas. O teste de Shapiro-Wilk foi utilizado para testar a normalidade e um p-valor >0,05 foi definido como dado normalmente distribuído. Variáveis contínuas que apresentaram distribuição normal foram comparadas utilizando-se o teste T de Student e ANOVA, enquanto o teste $U$ de Mann-Whitney e o teste de Kruskal-Wallis foram usados para amostras não distribuídas normalmente. Variáveis categóricas e frequências foram comparadas pelo teste Qui-quadrado. A significância estatística foi definida como um $p$-valor $<0,05$ para todas as comparações. Neste estudo, os parâmetros que foram diferentes em pacientes com $<20 \%$ para SLG-VE foram encontrados em análise univariada. Portanto, retroativamente: A análise de regressão logística (RL) foi realizada para se determinar os parâmetros que determinaram de forma independente pacientes com SLG-VE $<20 \%$. As correlações de Pearson e Spearman foram usadas para examinar a relação entre variáveis contínuas. Variáveis com um p-valor $<0,05$ na análise bivariada foram testadas na análise de regressão linear. Os resultados foram expressos como p-valor e razão de risco (RR) no IC de 95\%.

\section{Resultados}

Foram incluídos no estudo cinquenta e oito pacientes com acromegalia ativa. Foram excluídos do estudo oito pacientes que atenderam aos critérios de exclusão e que não puderam realizar o exame ecocardiográfico ideal. Dos pacientes incluídos no estudo, 45 eram pacientes recorrentes de acromegalia. Dos demais pacientes, três eram pacientes pós-cirurgia sem remissão, e dois estavam fazendo tratamento médico sem remissão. Os dados do estudo foram divididos em dois grupos, com e sem acromegalia (pacientes de controle saudáveis). Os coeficientes kappa de Cohen que avaliaram a variabilidade interobservador e intraobservador estavam acima de $90 \%$ de todas as medições de ecocardiogramas.

\section{Dados demográficos, clínicos e laboratoriais}

Quando os dados demográficos foram comparados entre os grupos do estudo: idade, sexo, IMC e ASC eram semelhantes entre os grupos. Determinou-se que a frequência de HT e DM em pacientes com acromegalia era de $28 \%$ e $32 \%$, respectivamente. Em termos de parâmetros clínicos, as pressões arteriais sistólica e diastólica, e a frequência cardíaca eram mais altas em pacientes com acromegalia. Identificouse que os níveis de glicemia plasmática, NT-proBNP, IGF-1 e hormônio de crescimento eram significativamente mais altos em pacientes com acromegalia. Parâmetros de outros laboratórios eram semelhantes entre os dois grupos (Tabela 1).

\section{Dados ecocardiográficos}

Identificou-se que os valores de SIV, espessura diastólica final da PP, e IMVE eram significativamente mais altos em pacientes com acromegalia (Tabela 2). Determinou-se que os diâmetros de VE e os valores de FEVE eram semelhantes entre os grupos com e sem acromegalia. Os valores de SAE e SLG-VE são significativamente mais baixos em pacientes com acromegalia (Figura 4-5). 


\begin{tabular}{|c|c|c|c|}
\hline Variável & $\begin{array}{l}\text { Pacientes com acromegalia } \\
\qquad n=50\end{array}$ & $\begin{array}{l}\text { Controles saudáveis } \\
\qquad n=50\end{array}$ & $\mathbf{p}$ \\
\hline Idade (anos) & $46,1 \pm 6,2$ & $44,6 \pm 5,1$ & 0,295 \\
\hline Sexo (feminino) & 17 & 19 & 0,418 \\
\hline Hipertensão, n (\%) & $14(28 \%)$ & - & - \\
\hline Diabetes mellitus, $n(\%)$ & $16(32 \%)$ & - & - \\
\hline Fumante, n (\%) & $15(30 \%)$ & - & - \\
\hline Hiperlipidemia, n (\%) & $7(14 \%)$ & - & - \\
\hline Pressão arterial sistólica $(\mathrm{mmHg})$ & $130 \pm 19$ & $110 \pm 10$ & $<0,001$ \\
\hline Pressão arterial diastólica $(\mathrm{mmHg})$ & $81 \pm 11$ & $67 \pm 6,4$ & $<0,001$ \\
\hline Frequência cardíaca (pulsos/minuto) & $81 \pm 11$ & $67 \pm 4,1$ & $<0,001$ \\
\hline Índice de massa corporal $\left(\mathrm{kg} / \mathrm{m}^{2}\right)$ & $28,1 \pm 2,3$ & $27,6 \pm 1,6$ & 0,164 \\
\hline Área da superfície corporal $\left(\mathrm{m}^{2}\right)$ & $2,01 \pm 0,10$ & $2,00 \pm 0,09$ & 0,569 \\
\hline Leucócito $(\mu \mathrm{L})$ & $7,3 \pm 1,9$ & $7,5 \pm 1,6$ & 0,656 \\
\hline Hemoglobina (gr/dL) & $13,1 \pm 1,8$ & $12,9 \pm 1,2$ & 0,420 \\
\hline Plasma glicemia (mg/dL) & $109 \pm 23$ & $92 \pm 5,6$ & $<0,001$ \\
\hline Nitrogênio ureico sanguíneo (mg/dL) & $32,9 \pm 16,6$ & $29,5 \pm 4,1$ & 0,149 \\
\hline Creatinina (mg/dL) & $0,75 \pm 0,42$ & $0,64 \pm 0,10$ & 0,138 \\
\hline Colesterol total $(\mathrm{mg} / \mathrm{dL})$ & $197 \pm 59$ & $217 \pm 60$ & 0,095 \\
\hline Lipoproteína de baixa densidade (mg/dL) & $135 \pm 45$ & $148 \pm 44$ & 0,157 \\
\hline Lipoproteína de alta densidade (mg/dL) & $44,3 \pm 15,3$ & $48,2 \pm 8,1$ & 0,125 \\
\hline Triglicérides (mg/dL) & $165 \pm 77$ & $191 \pm 108$ & 0,180 \\
\hline Aspartato aminotransferase $(\mathrm{u} / \mathrm{L})$ & $20,6 \pm 7,4$ & $18,9 \pm 3,4$ & 0,143 \\
\hline NT-proBNP (pg/mL) & $365 \pm 297$ & $74 \pm 6,7$ & $<0,001$ \\
\hline PCR-as (mg/dL) & $1,69 \pm 1,35$ & $0,43 \pm 0,31$ & $<0,001$ \\
\hline Alanina aminotransferase ( $u / L)$ & $16,8 \pm 8,9$ & $15,9 \pm 2,9$ & 0,298 \\
\hline IGF-1 (ng/dL) & $376 \pm 181$ & $72 \pm 7,5$ & $<0,001$ \\
\hline Hormônio do crescimento $(\mathrm{ng} / \mathrm{mL})$ & $9,21 \pm 14,4$ & $1,01 \pm 0,52$ & $<0,001$ \\
\hline
\end{tabular}

PCR-as: Proteina C reativa alta sensivel; IGF-1: Fator de crescimento semelhante à insulina tipo 1; NT-proBNP: Peptídeo natriurético pró-cérebro N-terminal.

Tabela 2 - Parâmetros ecocardiográficos de pacientes com acromegalia em pacientes de controle saudáveis

\begin{tabular}{lccc}
\hline Variável & $\begin{array}{c}\text { Pacientes com acromegalia } \\
\mathbf{n = 5 0}\end{array}$ & $\begin{array}{c}\text { Controles saudáveis } \\
\mathbf{n}=\mathbf{5 0}\end{array}$ & $\mathbf{p}$ \\
\hline Espessura diastólica final de SIV $(\mathrm{mm})$ & $12,3 \pm 1,92$ & $9,9 \pm 1,21$ & $<0,001$ \\
\hline Espessura diastólica final de PP $(\mathrm{mm})$ & $11,9 \pm 1,32$ & $9,7 \pm 1,01$ & $<0,001$ \\
\hline Dimensão diastólica final do VE $(\mathrm{mm})$ & $46,7 \pm 4,5$ & $47,3 \pm 4,3$ & 0,516 \\
\hline Dimensão sistólica final do VE $(\mathrm{mm})$ & $31,1 \pm 4,2$ & $31,5 \pm 4,2$ & 0,656 \\
\hline Dimensão diastólica final do AE $(\mathrm{mm})$ & $35,3 \pm 4,2$ & $33,1 \pm 2,6$ & 0,002 \\
\hline Fração de ejeção VE $(\%)$ & $57,8 \pm 4,1$ & $58,9 \pm 5,3$ & 0,259 \\
\hline Índice de massa do VE $\left(\mathrm{gr} / \mathrm{m}^{2}\right)$ & $108 \pm 28$ & $82 \pm 17$ & $<0,001$ \\
\hline Hipertrofia do VE, $\mathrm{n}(\%)$ & $25(50 \%)$ & $0(0 \%)$ & $<0,001$ \\
\hline SAE $(\%)$ & $21,5 \pm 1,36$ & $23,5 \pm 1,06$ & $<0,001$ \\
\hline SLG-VE $(\%)$ & $-20,4 \pm 1,45$ & $-22,8 \pm 0,83$ & $<0,001$ \\
\hline SLG-VE $<20, \mathrm{n}(\%)$ & $24(48 \%)$ & $0(0 \%)$ & $<0,001$ \\
\hline
\end{tabular}

SIV: Septo interventricular; AE: Átrio esquerdo; SLG-VE: Deformação longitudinal global do ventrículo esquerdo; VE: ventrículo esquerdo; PP: Parede posterior; SAE: deformação de AE no pico positivo. 


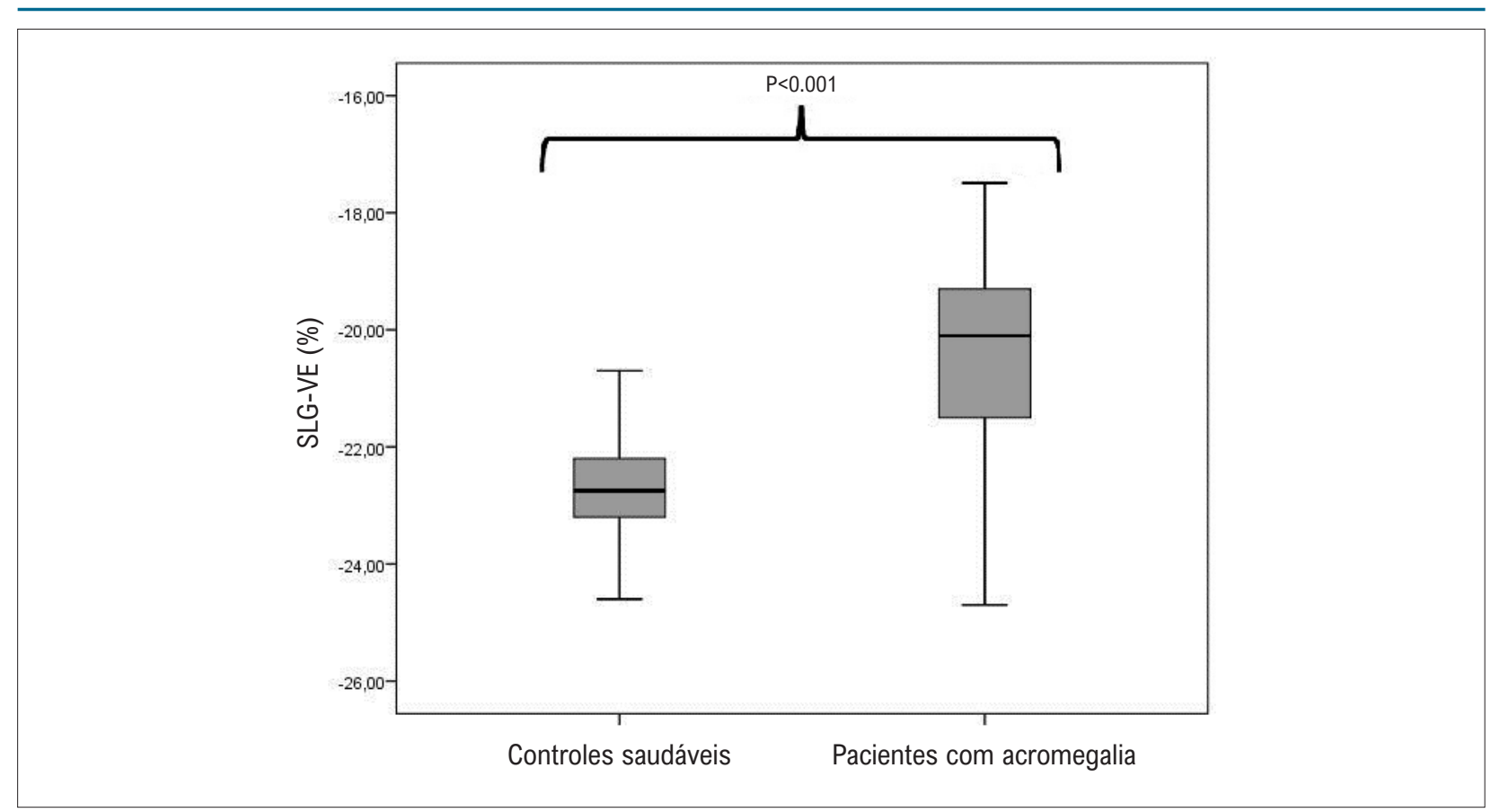

Figura 4 - 0 gráfico de caixa demonstrou a deformação longitudinal global do ventrículo esquerdo (SLG-VE) em pacientes com acromegalia e em pacientes de controle saudáveis.

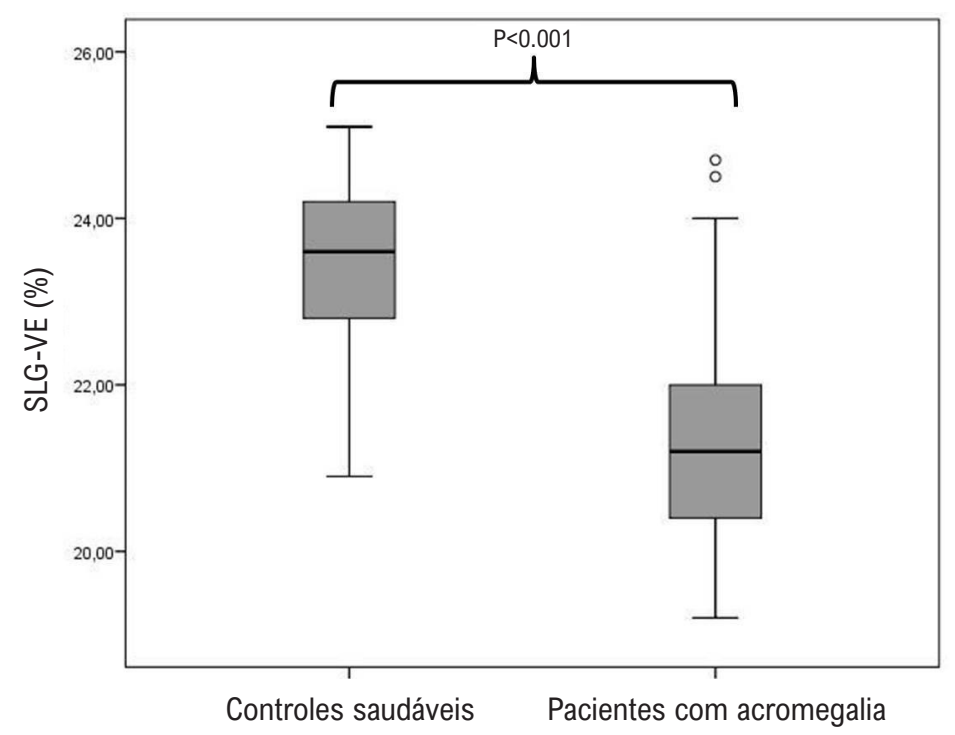

Figura 5-O gráfico de caixa demonstrou o pico positivo de deformação de AE (SAE) em pacientes com acromegalia e em pacientes de controle saudáveis.

Quando o valor limite foi tomado como $<20 \%$ para SLGVE, detectou-se que os níveis de SLG-VE eram reduzidos em $48 \%$ do grupo com acromegalia. Quando os dados demográficos, clínicos e laboratoriais de pacientes com acromegalia SLG-VE normal e reduzida foram comparados, determinou-se que pacientes com SLG-VE baixo tinham índices mais altos de HT $(45,8 \%$ x 11,5\% e p = 0,008) e valores mais altos de IMVE $\left(123 \mathrm{gr} / \mathrm{m}^{2}\right.$ x 94,6 gr/m² e $\left.\mathrm{p}<0,008\right)$. $\mathrm{Na}$ análise de regressão logística multivariada, detectou-se que o valor de IMVE e os níveis de IGF-1 fazem a previsão independente do valor de SLG-VE reduzido $(p=0,003$, RC: 1,060 e IC: 1,019 - 1,102 e $p=0,012$, RC: 1,056 e IC: 1,023 - 1,098). De acordo com essa análise, cada aumento de $1 \mathrm{gr} / \mathrm{m}^{2}$ no valor de IMVE e cada aumento de $1 \mathrm{ng} / \mathrm{dL}$ nos 
níveis de IGF-1 aumenta a probabilidade de diminuição de SLG-VE em 6\% e 5,6\%, respectivamente.

\section{Determinação da medição de deformação do AE}

A análise de correlação foi realizada para determinar os parâmetros associados à deformação do AE. Os parâmetros relacionados a SAE na análise de correlação foram resumidos na Tabela 3. A análise de regressão linear foi realizada para determinar a presença de relações independentes de SAE. $\mathrm{Na}$ análise de regressão linear, detectou-se uma associação positiva e significativa entre PA sistólica, NT-proBNP, IGF-1, diâmetro diastólico final do AE e IMVE, e SAE. Estatisticamente, a correlação mais forte identificada foi entre SAE e os níveis de IGF-1 (Tabela 3 e Figura 6).

\section{Determinação de parâmetros relacionados a SLG-VE}

A análise de correlação foi realizada para determinar os parâmetros associados à deformação do SLG-VE. Os parâmetros relacionados a SLG-VE na análise de correlação foram resumidos na Tabela 4. A análise de regressão linear foi realizada para determinar a presença de relações independentes de parâmetros relacionados a SLG-VE. Na análise de regressão linear, detectou-se uma associação positiva e significativa entre pressão arterial sistólica, NTproBNP, IGF-1, diâmetro diastólico final do AE e IMVE, e valor de SLG-VE. Estatisticamente, a correlação mais forte identificada foi entre SLG-VE e os níveis de IGF-1 (Tabela 4 e Figura 7).

\section{Discussão}

Até onde sabemos, este estudo é primeiro estudo a avaliar SLG-VE e SAE juntos em pacientes com acromegalia. O principal achado deste estudo foi a determinação de que as funções sistólicas do VE e do AE em ES eram prejudicadas apesar da preservação das funções sistólicas do VE no ecocardiograma convencional. Outro achado importante foi que o nível de IGF-1, que era um dos parâmetros mais importantes da atividade da doença acromegalia, tinha uma forte correlação com o SAE e o SLG-VE. Além disso, neste estudo, identificou-se que $48 \%$ dos pacientes com a acromegalia tinham uma deficiência silenciosa da função sistólica do VE detectada pelo ES, e essa condição foi próxima e independentemente relacionada ao valor de IMVE e nível de IGF-1.

A acromegalia é uma das causas secundárias do HT. A HT e a DM são comuns nesses pacientes devido aos efeitos metabólicos da doença. 7,11,21,22 Em pacientes com acromegalia, fibrose e alterações hipertróficas ocorrem em graus diferentes miocárdio de $\mathrm{AE}$ e $\mathrm{VE}$, sendo que ambos aumentam a frequência de HT e DM, e o nível de IGF-1. A HVE é comum e pode ser vista em 25-85\% dos pacientes com acromegalia. ${ }^{5,23,24}$ Neste estudo, $50 \%$ dos pacientes com acromegalia tinham HVE. Em pacientes com acromegalia, as alterações cardíacas devidas ao aumento de hormônios e HT e DM associadas são chamadas miocardiopatia acromegálica (CMP). ${ }^{25}$ A doença consiste em 3 fases: i) aumento da contratilidade do VE e do ventrículo direito (VD) e HVE acompanhado do aumento da IGF-1, ii) disfunção diastólica resultante de redução da elasticidade do VE, iii) aparência típica de cardiomiopatia com dilatação do VE e diminuição de FEVE. ${ }^{26}$ A diminuição de FEVE e dilatação do VE, que são o terceiro estágio da doença, são vistas apenas em 1 a $10 \%$ dos pacientes com acromegalia. ${ }^{11-14}$ Entretanto, é importante avaliar as funções do VE desses pacientes com um novo método diagnóstico já desde o estágio 1, antes da ocorrência de alterações cardíacas irreversíveis.

A avaliação da função sistólica do VE com SLG-VE é relativamente nova e ainda não é muito comum. O SLG-VE é mais utilizado para avaliar o envolvimento cardíaco subclínico ou silencioso de doenças sistêmicas com FEVE normal..$^{27-30}$ A mortalidade cardiovascular aumenta em pacientes com acromegalia. ${ }^{1,2}$ Foi demonstrado que os valores de SLG-VE reduzidos estão associados a morte súbita cardíaca e arritmia potencialmente fatal. ${ }^{31} \mathrm{O}$ fato de que o ES é facilmente acessível e barato dá uma vantagem importante em relação

Tabela 3 - Os parâmetros associados ao SLG-AE e à análise por regressão linear para parâmetros significativamente correlacionados a SAE

\begin{tabular}{|c|c|c|c|c|}
\hline & \multicolumn{2}{|c|}{ Análise univariada } & \multicolumn{2}{|c|}{ Análise multivariada } \\
\hline & $\mathbf{p}$ & $\mathbf{r}$ & $\mathbf{p}$ & $\beta$ \\
\hline Pressão arterial sistólica & $<0,001$ & 0,427 & 0,001 & 0,278 \\
\hline Pressão arterial diastólica & $<0,001$ & 0,362 & 0,470 & 0,102 \\
\hline Frequência cardíaca & $<0,001$ & 0,360 & 0,840 & 0,023 \\
\hline Glicemia plasmática & $<0,001$ & 0,418 & 0,255 & 0,133 \\
\hline Creatinina & 0,018 & 0,225 & 0,712 & 0,064 \\
\hline NT-proBNP & $<0,001$ & 0,445 & 0,013 & 0,237 \\
\hline IGF-1 (ng/dL) & $<0,001$ & 0,531 & $<0,001$ & 0,531 \\
\hline Hormônio do crescimento (ng/mL) & 0,025 & 0,225 & 0,408 & 0,096 \\
\hline Dimensão diastólica final do $A E$ & $<0,001$ & 0,662 & $<0,001$ & 0,378 \\
\hline Índice de massa do VE & $<0,001$ & 0,623 & $<0,001$ & 0,503 \\
\hline
\end{tabular}

AE: Átrio esquerdo; SAE: deformação de AE no pico positivo; VE: Ventrículo esquerdo; IGF-1: Fator de crescimento semelhante à insulina tipo 1; NT-proBNP:

Peptídeo natriurético pró-cérebro $N$-terminal. $R_{\text {padronizado }}^{2}=0,684$ e $p<0.001$ análises multivariadas. 


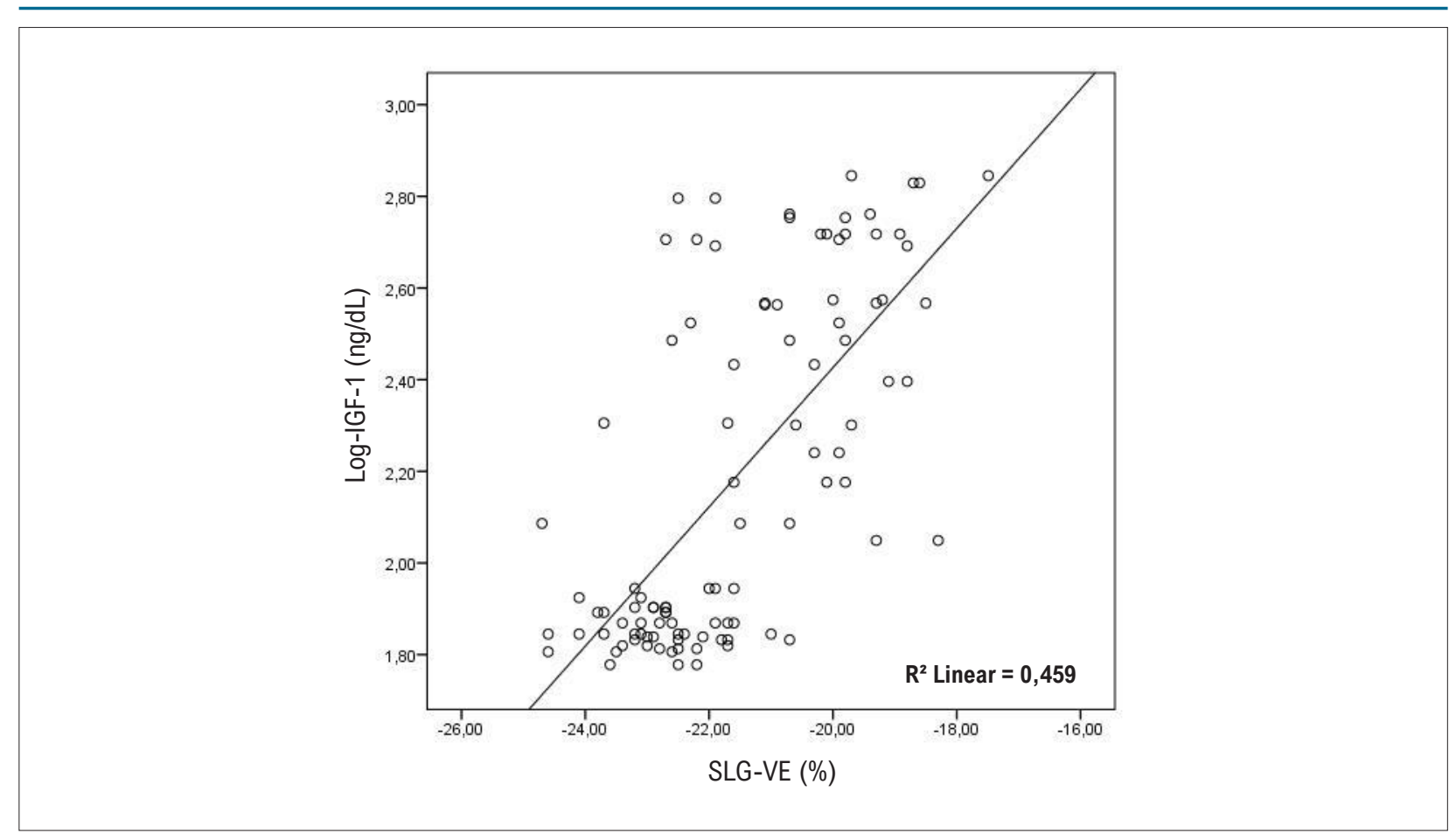

Figura 6 - Gráfico de dispersão da relação do pico positivo de deformação de AE (SAE) com fator de crescimento semelhante à insulina tipo 1 (IGF-1). Foi obtida uma escala logaritmica na base 10 de IGF-1.

Tabela 4 - Os parâmetros associados ao SLG-VE e à análise por regressão linear para parâmetros significativamente correlacionados a SLG-VE

\begin{tabular}{lcccc}
\hline & \multicolumn{2}{c}{ Análise univariada } & \multicolumn{2}{c}{ Análise multivariada } \\
\cline { 2 - 5 } & $\mathbf{p}$ & $\mathbf{r}$ & $\mathbf{p}$ & $\boldsymbol{\beta}$ \\
\hline Idade & 0,026 & 0,223 & 0,844 & 0,017 \\
\hline Índice de massa corporal & 0,033 & 0,213 & 0,256 & 0,092 \\
\hline Pressão arterial sistólica & $<0,001$ & 0,509 & $<0,001$ & 0,300 \\
\hline Pressão arterial diastólica & $<0,001$ & 0,462 & 0,605 & 0,076 \\
\hline Frequência cardíaca & $<0,001$ & 0,408 & 0,426 & 0,081 \\
\hline Glicemia plasmática & $<0,001$ & 0,442 & 0,172 & 0,146 \\
\hline Creatinina & 0,015 & 0,243 & 0,263 & 0,090 \\
\hline NT-proBNP & $<0,001$ & 0,478 & 0,011 & 0,176 \\
\hline IGF-1 & $<0,001$ & 0,626 & $<0,001$ & 0,626 \\
\hline Hormônio do crescimento $(\mathrm{ng} / \mathrm{mL})$ & $<0,001$ & 0,429 & 0,050 & 0,207 \\
\hline Dimensão diastólica final do AE & 0,001 & 0,009 & 0,199 \\
\hline Índice de massa do VE & $<0,001$ & $<0,001$ & 0,548 \\
\hline
\end{tabular}

IGF-1: Fator de crescimento semelhante à insulina tipo 1; NT-proBNP: Peptídeo natriurético pró-cérebro N-terminal; SLG-VE: Deformação longitudinal global do ventrículo esquerdo. $R_{\text {padronizado }}^{2}=0,641$ e $p<0.001$ análises multivariadas.

aos outros métodos. Entretanto, a limitação mais importante é que a qualidade da imagem deve ser muito boa. Embora o ES seja usado frequentemente para a avaliação inicial das funções do VE; nos últimos anos, muitos estudos avaliaram as funções do AE com ecocardiograma strain. ${ }^{4}$

Vários estudos avaliaram o SLG-VE em pacientes com acromegalia e FEVE normal e foram obtidos resultados contraditórios. ${ }^{5-7} \mathrm{O}$ primeiro estudo foi realizado por Volschan et al. ${ }^{5}$, em 2017, em 37 pacientes com acromegalia ativa, e detectou-se que o valor de SLG-VE não variou nem aumentou sem significância estatística em comparação aos pacientes de controle. Em outro estudo realizado em 2018, relatou-se que houve uma diminuição no valor do SLG-VE nos pacientes com acromegalia e essa situação estava relacionada a HVE. ${ }^{7}$ Em 


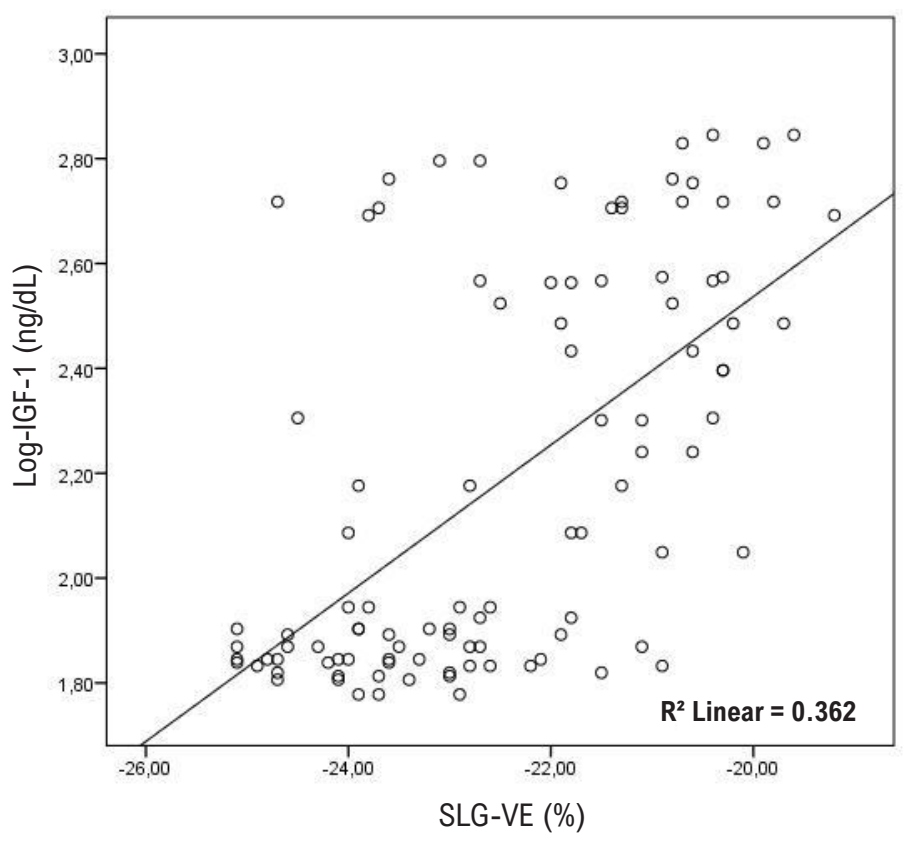

Figura 7 - Gráfico de dispersão da relação da deformação longitudinal global do ventrículo esquerdo (SLG-VE) com fator de crescimento semelhante à insulina tipo 1 (IGF-1). Foi obtida uma escala logarítmica na base 10 de IGF-1.

outro estudo, realizado e publicado muito recentemente pelos mesmos autores, relatou-se que o SLG-VE era reduzido em pacientes com acromegalia, da mesma forma que o estudo anterior. ${ }^{6}$ Este estudo corrobora dois estudos que demonstram que o valor de SLG-VE é reduzido em pacientes com acromegalia. Além dos estudos anteriores, também demonstramos uma redução significativa no SLGAE no mesmo grupo de pacientes. O valor de corte para o SLG-VE reduzido foi aceito como $<20 \% .^{15}$ Neste estudo, $48 \%$ dos pacientes com acromegalia ficavam $<20 \%$. Em outras palavras, as funções sistólicas do VE de metade dos pacientes deste estudo com acromegalia estavam comprometidas. Identificou-se que o IMVE e o IGF-1 tinham uma forte associação com o SLG-VE reduzido em pacientes com acromegalia. ${ }^{5,7}$ Os níveis de GH e IGF-1 sérico não estavam relacionados em pacientes com SLGVE reduzido nos mesmos estudos. ${ }^{5,7}$ Neste estudo, assim como no estudo anterior, identificou-se que o IMVE previa pacientes com SLG-VE reduzido. Além disso, os níveis de IGF-1 estavam significativamente relacionados ao SLG-VE reduzido. Neste estudo, identificou-se que cada aumento de $1 \mathrm{gr} / \mathrm{m}^{2}$ de IMVE aumentava o risco de SLG-VE reduzido em $6 \%$. Além disso, cada aumento de $1 \mathrm{ng} / \mathrm{dL}$ nos níveis de IGF-1 aumenta a probabilidade de diminuição de SLGVE em 5,6\%. O IMVE, que é o achado mais objetivo do envolvimento cardíaco em pacientes com acromegalia, também é o parâmetro mais proximamente associado ao SLG-VE reduzido. Portanto, a intervenção de HT, DM e IMVE o mais cedo possível pode ser a forma mais lógica de retardar a disfunção sistólica futura em pacientes com acromegalia.
O SLG-VE comprometido em pacientes com acromegalia pode ser explicado por dois mecanismos fisiopatológicos. O primeiro é o efeito de HT e DM. A redução do SLG-VE foi demonstrada anteriormente com os efeitos cardíacos de HT e DM mesmo no período assintomático antes de qualquer doença $\mathrm{CV}^{27,28}$ Determinou-se que a frequência de HT e DM em pacientes com acromegalia era de $28 \%$ e $32 \%$, respectivamente. A prevalência de HT foi significativamente mais alta em pacientes com SLG-VE reduzido. Isso indica que o SLG-VE é afetado pela presença de HT. O segundo mecanismo pode ser a heterotrofia do VE e fibrose miocárdica devido ao aumento do IGF-1 em pacientes com acromegalia sem HT e DM. ${ }^{23,25} \mathrm{O}$ aumento do valor de IGF-1 pode estar associado à atividade da doença e envolvimento cardíaco. Neste estudo, o nível de IGF-1, que era um dos parâmetros mais importantes da atividade da doença acromegalia, tinha uma forte correlação com o SLG-VE.

Há dados limitados sobre a função e o tamanho do AE em pacientes com acromegalia. ${ }^{8,32} \mathrm{Em}$ um estudo anterior, relatou-se que o volume e as funções mecânicas do AE eram semelhantes aos dos pacientes de controle saudáveis e os níveis de GH e IGF-1 séricos não foram associados à função mecânica do AE em pacientes com acromegalia. ${ }^{32}$ Em outro estudo recente, um aumento no volume do AE foi relatado em pacientes com acromegalia. ${ }^{8}$ Em nosso estudo, o volume do AE ainda não foi avaliado, mas o diâmetro diastólico do AE era aumentado em pacientes com acromegalia.

Embora não haja estudos na literatura avaliando o SAE com 2D-STE em pacientes com acromegalia, as imagens da deformação de AE foram realizadas em apenas um estudo com 3 D-STE. ${ }^{8}$ Kormanyos et al. ${ }^{8}$ relataram uma redução 
nos valores de deformação segmentar médios e globais, e uma redução no valor de deformação circunferencial de AE. Um achado semelhante foi demonstrado para o átrio direito em outro estudo pelos mesmos autores. ${ }^{33}$ Relatou-se que o nível de IGF-1 e o valor de deformação circunferencial do AE tinham uma correlação positiva. ${ }^{8}$ Nosso estudo foi o primeiro a demonstrar a redução do SAE e sua correlação forte e positiva com o IGF-1, que é um dos parâmetros de atividade da doença.

\section{Limitações}

Como um estudo não randomizado de centro único, esta coorte de pacientes pode ser diferente da de outros centros. O tamanho da amostra é relativamente pequeno e os resultados do estudo precisam ser confirmados ensaios prospectivos multicêntricos grandes no futuro. A mortalidade e a morbidade cardiovasculares são altas em pacientes com acromegalia. ${ }^{1}$ Entretanto, os prognósticos não foram avaliados. Além disso, o efeito do tratamento de SLG-VE e SAE não foi avaliado devido à ausência de um acompanhamento. Neste estudo, não tivemos informações sobre o insight fisiopatológico do SLG-VE reduzido devido à ausência de avaliação histopatológica com biópsia miocárdica. A apneia do sono é comum em pacientes com acromegalia, ${ }^{1}$ e tem um efeito adverso nas funções de VE. Entretanto, não foi possível fazer a polissonografia em todos os pacientes. Os tipos de envolvimento cardíaco mais importantes são HVE e fibrose miocárdica em pacientes com acromegalia. A fibrose miocárdica é mais bem avaliada por imagens por ressonância magnética cardíaca. Se tivesse sido possível realizar as imagens por ressonância magnética cardíaca, teria sido possível avaliar a associação entre realce tardio pelo gadolínio e SLG-VE e SLG-AE. A disfunção diastólica também era comum (50,5\%) em pacientes com acromegalia. ${ }^{34}$ Portanto, não avaliamos a disfunção diastólica nesses grupos de pacientes.

Estudos anteriores demonstraram que o volume do VE e do AE e o índice de volume aumentou em pacientes com acromegalia. ${ }^{6,8}$ Neste estudo, foram medidos apenas o diâmetro do VE e o diâmetro diastólico final do AE. Se o

\section{Referências}

1. Katznelson L, Laws ER Jr, Melmed S, Molitch ME, Murad MH, Utz A, et al. Endocrine Society. Acromegaly: an endocrine society clinical practice guideline. J Clin Endocrinol Metab. 2014;99:39(4)33-51.

2. Gondim JA, Almeida JP, de Albuquerque LA, Gomes E, Schops M, Ferraz T. Pure endoscopic transsphenoidal surgery for treatment of acromegaly: results of 67 cases treated in a pituitary center. Neurosurg Focus. 2010;29(4):E7.

3. Tops LF, Delgado V, Marsan NA, Bax JJ. Myocardial strain to detect subtle left ventricular systolic dysfunction. Eur J Heart Fail. 2017; 19(3): 307-13.

4. Koca H, Demirtas AO, Kaypaklı O, Icen YK, Sahin DY, Koca F, etal. Decreased left atrial global longitudinal strain predicts the risk of atrial fibrillation recurrence after cryoablation in paroxysmal atrial fibrillation. J Interv Card Electrophysiol. 2020 Jun;58(1):51-9.

5. Volschan ICM, Kasuki L, Silva CMS, Alcantara ML, Saraiva RM, Xavier SS, et al. Two-dimensional speckle tracking echocardiography demonstrates no effect of active acromegaly on left ventricular strain. Pituitary. 2017 Jun;20(3):349-57. índice de volume do AE e do VE também tivesse sido medido, poderia haver alterações, especialmente nos parâmetros relacionados a SLG-AE.

\section{Conclusão}

Embora a FEVE seja normal em pacientes com acromegalia, SAE e SLG-VE detectados com 2D-STE são significativamente reduzidos e têm uma relação próxima aos níveis de IGF-1 plasmático. Além do aumento em IMVE, outro achado do envolvimento cardíaco pode ser a redução de SAE e SLG-VE. Portanto, além do ecocardiograma de rotina, SAE e SLG-VE podem ser úteis para avaliar os sinais iniciais de envolvimento cardíaco antes da ocorrência de alterações cardíacas irreversíveis em pacientes com acromegalia.

\section{Contribuiç̧ão dos autores}

Concepção e desenho da pesquisa e Análise estatística: Koca K, Koc M, Icen YK, Baykan AO, Kaypakli O; Obtenção de dados: Sumbul HE, Icen YK, Gulumsek E, Koca F, Ozturk HA; Análise e interpretação dos dados: Koca K, Gulumsek E, Koca F, Ozturk HA; Redação do manuscrito: Koca K, Koc M, Koca F, Baykan AO, Kaypakli O; Revisão crítica do manuscrito quanto ao conteúdo intelectual importante: Koca K, Sumbul $\mathrm{HE}$, Kaypakli O.

\section{Potencial conflito de interesse}

Não há conflito com o presente artigo

\section{Fontes de financiamento}

O presente estudo não teve fontes de financiamento externas.

\section{Vinculação acadêmica}

Não há vinculação deste estudo a programas de pósgraduação.

6. Popielarz-Grygalewicz A, Stelmachowska-Banaś M, Gąsior JS, Grygalewicz P, Czubalska M, Zgliczyński W, et al. Subclinical left ventricular systolic dysfunction in patients with naive acromegaly - assessment with twodimensional speckle-tracking echocardiography: retrospective study. Endokrynol Pol. 2020 Apr 15. doi: 10.5603/EP.a2020.0021.

7. Popielarz-Grygalewicz A, Gąsior JS, Konwicka A, Grygalewicz P, Stelmachowska-Banaś M, Zgliczyński W, et al. Heart in Acromegaly: The Echocardiographic Characteristics of Patients Diagnosed with Acromegaly in Various Stages of the Disease. Int J Endocrinol. 2018 Jul 11;2018:6935054.

8. Kormányos Á, Domsik P, Kalapos A, Valkusz Z, Lengyel C, Forster T, et al. Three-dimensional speckle tracking echocardiography-derived left atrial deformation analysis in acromegaly (Results from the MAGYAR-Path Study). Echocardiography. 2018 Jul;35(7):975-84.

9. Varghese MJ, Sharma G, Shukla G, Seth S, Mishra S, Gupta A, et al. Longitudinal ventricular systolic dysfunction in patients with very severe obstructive sleep apnea: A case control study using speckle tracking imaging. Indian Heart J. 2017;69(3):305-10. 
10. Uziębło-Życzkowska B, Krzesinński P, Witek P, Zielinński G, JurekA, Gielerak G, et al. Cushing's Disease: Subclinical Left Ventricular Systolic and Diastolic Dysfunction Revealed by Speckle Tracking Echocardiography and Tissue Doppler Imaging. Front Endocrinol (Lausanne). 2017; 8 Sept 5: 222.

11. Mosca S, Paolillo S, Colao A, Bossone E, Cittadini A, ludice FL, et al. Cardiovascular involvement in patients affected by acromegaly: an appraisal. Int J Cardiol. 2013; 167(5):1712-8.

12. Colao A, Cuocolo A, Marzullo P, Nicolai E, Ferone D, Della Morte AM, et al. Impact of patient's age and disease duration on cardiac performance in acromegaly: a radionuclide angiography study. J Clin Endocrinol Metab. 1999; 84(5):1518-23.

13. Colao A, Cuocolo A, Marzullo P, Nicolai E, Ferone D, Della Morte AM, et al. Is the acromegalic cardiomyopathy reversible? Effect of 5-Year normalization of growth hormone and insulin-like growth factor I levels on cardiac performance. J Clin Endocrinol Metab. 2001; 86(4):1551-7.

14. Bihan H, Espinosa C, Valdes-Socin H, Salenave S, Young J, Levasseur S, et al. Longterm outcome of patients with acromegaly and congestive heart failure. J Clin Endocrinol Metab. 2004;89(11):5308-13.

15. Lang RM, Badano LP, Mor-Avi V, Afilalo J, Armstrong A, Ernande L, et al. Recommendations for cardiac chamber quantification by echocardiography in adults: an update from the American Society of Echocardiography and the European Association of Cardiovascular Imaging. J Am Soc Echocardiogr $.2015 ; 28(1): 1-39 . e 14$

16. Devereux RB, Alonso DR, Lutas EM, Gottlieb GJ, Campo E, Sachs I, et al. Echocardiographic assessment of left ventricular hypertrophy: comparison to necropsy findings. Am J Cardiol. 1986;57(6):450-8.

17. Nagueh SF, Smiseth OA, Appleton CP, Byrd BF 3rd, Dokainish H, Edvardsen $\mathrm{T}$, et al. Recommendations for the Evaluation of Left Ventricular Diastolic Function by Echocardiography: An Update from the American Society of Echocardiography and the European Association of Cardiovascular Imaging. J Am Soc Echocardiogr. 2016; 29(4): 277-314

18. Voigt JU, Pedrizzetti G, Lysyansky P, Marwick TH, Houle H, Baumann $R$, et al. Definitions for a common standard for 2D speckle tracking echocardiography: consensus document of the EACVI/ASE/Industry Task Force to standardize deformation imaging. Eur Heart J Cardiovasc Imaging. 2015 Jan;16(1):1-11.

19. Badano LP, Kolias TJ, Muraru D, Abraham TP, Aurigemma G, Edvardsen $\mathrm{T}$, et al. Standardization of left atrial, right ventricular, and right atrial deformation imaging using two-dimensional speckle tracking echocardiography: a consensus document of the EACVI/ASE/Industry Task Force to standardize deformation imaging. Eur Heart J Cardiovasc Imaging. 2018 Jun 1;19(6):591-600.

20. Donal E, Galli E, Schnell F. Left Atrial Strain: A Must or a Plus for Routine Clinical Practice? Circ Cardiovasc Imaging. 2017 Oct; 10(10):e007023.

21. Møller J, Jørgensen JO, Marqversen J, Frandsen E, Christiansen JS. Insulinlike growth factor I administration induces fluid and sodium retention in healthy adults: possible involvement of renin and atrial natriuretic factor. Clin Endocrinol (Oxf). 2000;52(2):181-6.

22. Mancia G, Fagard R, Narkiewicz K, Redon J, Zanchetti A, Böhm M, et al 2013 ESH/ESC Guidelines for the management of arterial hypertension: The Task Force for the Management of Arterial Hypertension of the European Society of Hypertension (ESH) and of the European Society of Cardiology (ESC). Eur Heart J. 2013 Jul;34(28):2159-219.

23. Lie JTG. Pathology of heart in acromegaly: anatomic findings in 27 autopsied patients. Am Heart J. 1980;100(1):41-52.

24. dos Santos Silva CM, Gottlieb I, Volschan I, Kasuki L, Warszawski L, Balarin Lima GA, et al. Low frequency of cardiomyopathy using cardiac magnetic resonance imaging in an acromegaly contemporary cohort. J Clin Endocrinol Metab. 2015;100(12):4447-55

25. Clayton RN. Cardiovascular function in acromegaly. Endocr Rev. 2003 Jun;24(3):272-7.

26. Reid TJ, Post KD, Bruce JN, Nabi Kanibir M, Reyes-Vidal CM, Freda PU Features at diagnosis of 324 patients with acromegaly did not change from 1981 to 2006: acromegaly remains under-recognized and under-diagnosed. Clin Endocrinol (Oxf). 2010 Feb;72(2):203-8.

27. Shin SM, Shim WJ, Park SM. Early changes of left ventricular function in young adults with never-treated hypertension and no left ventricular hypertrophy: relationships to ambulatory blood pressure monitoring. Clin Exp Hypertens. 2014;36(7):517-23.

28. Zairi I, Mzoughi K, Kamoun S, Moussa FB, Rezgallah R, Maatoug J, et al Impairment of left and right ventricular longitudinal strain in asymptomatic children with type 1 diabetes. Indian Heart J. 2019 May - Jun;71(3):249-55.

29. de Almeida AL, Gjesdal O, Mewton N, Choi EY, Teixido-Tura G, Yoneyama $\mathrm{K}$, et al. Speckle-Tracking pela Ecocardiografia Bidimensional - Aplicações Clínicas. Revista Brasileira Ecocardiogr imagem cardiovasc. 2013; 26:38-49.

30. Marwick TH, Venn AJ. The strain of detecting early target organ damage in hypertension. J Am Coll Cardiol. 2015;65(15):2688-90.

31. Ersboll M, Valeur N, Andersen MJ, Mogensen UM, Vinther M, Svendsen $\mathrm{JH}$, et al. Early echocardiographic deformation analysis for the prediction of sudden cardiac death and lifethreatening arrhythmias after myocardial infarction. JACC Cardiovasc Imaging. 2013;6(8):851-60.

32. Ilter A, Kiris A, Kaplan S, Kutlu M, Şahin M, Erem C, et al. Atrial conduction times and left atrium mechanical functions in patients with active acromegaly. Endocrine. 2015;48(2):653-60.

33. Kormányos Á, Kalapos A, Domsik P, Gyenes N, Ambrus N, Valkusz Z, et al. The right atrium in acromegaly-a three-dimensional speckle-tracking echocardiographic analysis from the MAGYAR-Path Study. Quant Imaging Med Surg. 2020;10(3):646-56.

34. Guo X, Fu H, Pang H, Xing B. Risk of left ventricular hypertrophy and diastolic and systolic dysfunction in Acromegaly: A meta-analysis. J Clin Neurosci. 2018 Feb;48:28-33 